\title{
Dynamic Balance is Similar Between Lower Extremities in Elite
}

\section{Fencers}

Nader Abdelkader', Stephen H.M. Brown'ㄹ, Tyson A.C. Beach ${ }^{3}$, Samuel J. Howarth ${ }^{1}$ a

1 Division of Research and Innovation, Canadian Memorial Chiropractic College, ${ }^{2}$ Department of Human Health and Nutritional Sciences, University of Guelph, ${ }^{3}$ Department of Kinesiology, University of Waterloo

Keywords: fencing, lower extremity, asymmetry, y-balance test

https://doi.org/10.26603/001c.29852

\section{International Journal of Sports Physical Therapy}

Vol. 16, Issue 6, 2021

\section{Background}

Few studies have quantified dynamic balance in fencers despite previous suggestions that balance training may be beneficial for these athletes. Generally, asymmetry in dynamic balance performance between the left and right legs can be an indicator of lower extremity injury risk and used to monitor rehabilitation progress. Fencing is recognized as an asymmetric sport, therefore, differences in dynamic balance may exist among uninjured athletes.

\section{Hypothesis/Purpose}

The primary objective of this investigation was to evaluate whether asymmetry of dynamic balance is present in uninjured national-level fencers. It was hypothesized that elite uninjured fencers would demonstrate superior dynamic balance on the lead-leg of their fencing stance. A secondary objective was to compare dynamic balance performance of elite fencers to previously published data from high-level athletes participating in other sports.

\section{Study Design}

Descriptive Laboratory Study

\section{Methods}

Fourteen uninjured elite competitive fencers were recruited. Subjects self-reported the lead leg of their fencing stance. Each participant performed the Y-Balance test (YBT), which represented a measurement of dynamic balance control, on both legs. Reach distances were recorded directly from a commercially available YBT apparatus. Four reach distances were recorded: anterior, posteromedial, posterolateral, and a composite measure was calculated. Distances were leg length-normalized and expressed as a percentage. Sample averages and standard deviations were derived for the four YBT measurements.

\section{Results}

There were no significant differences in reaching distance between the lead and trail legs in any of the four YBT measures $(p \geqslant 0.65)$. Fencers appeared to demonstrate larger normalized reach distances in the posterolateral and posteromedial directions than other

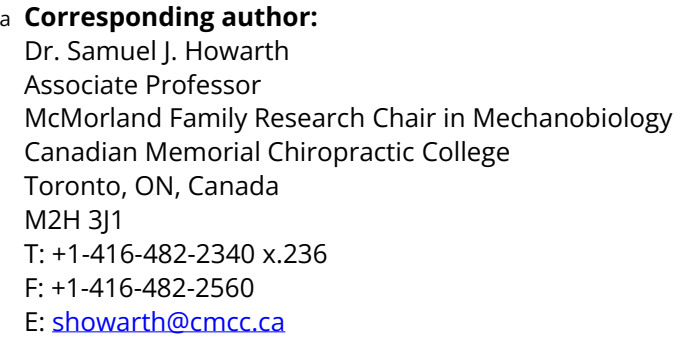


athletes.

\title{
Conclusions
}

The results of this study indicate that dynamic balance is not significantly different between the lead and trail legs in elite fencers, despite the asymmetrical nature of their sport. The apparent symmetry of dynamic balance control in uninjured fencers means that the YBT could be used in this population for monitoring progress during training and rehabilitation.

\section{Level of Evidence}

\author{
$2 \mathrm{~b}$
}

\section{INTRODUCTION}

Balance, and in particular dynamic balance, is recognized as a fundamental component of athletic performance. ${ }^{1}$ Dynamic balance is often operationally defined in athletic contexts by lower extremity reach distances on the Star Excursion Balance Test (SEBT) or the Y-Balance Test (YBT). 2,3 These tests measure a person's ability to actively control volitional movement during unipedal stance. Differences in performance between the left and right legs for a given reaching direction, hereafter referred to as asymmetries, can also be used as indicators of lower extremity injury risk and to monitor progress during rehabilitation from an injury. ${ }^{4-9}$ Previous authors have demonstrated that balance training and assessment may be beneficial for fencing performance; ${ }^{10,11}$ however, there has been limited research on balance performance in fencers. Furthermore, the presence of dynamic balance asymmetry (or lack thereof) in elite uninjured fencers, possibly related to sporting asymmetry, is unknown. A quantitative analysis of dynamic balance performance in elite competitive fencers, and comparison with data reported from elite athletes participating in other sports, is necessary before addressing recommendations related to training, rehabilitation monitoring, and lower extremity injury risk.

As previously mentioned, the literature on balance performance in fencers is limited. Herpin and colleagues investigated the influence of training on weighting of sensory inputs for balance control by comparing expert fencers to expert pistol shooters. ${ }^{12}$ Fencers were found to have an enhanced ability to integrate vestibular and proprioceptive information to control static balance than the pistol shooters. Another study used measures from static balance assessments to evaluate the effectiveness of a training program that addressed muscle imbalances in fencers. ${ }^{13}$ A possible limitation of these two studies was that the balance task did not require the participant to demonstrate dynamic balance control during volitional movements, which is required during fencing.

More recent studies have evaluated dynamic balance control in fencers with the aims of identifying possible asymmetries and changes following a proprioceptive training program. ${ }^{14,15}$ In the authors' opinion, the existing literature on balance assessment and training in fencers, implies that symmetry appears to be desirable. The extent to which the bilaterally asymmetric nature of fencing, attributable to the split stance adopted during competition, contributes to asymmetry during dynamic balance assessment is likely an important consideration. ${ }^{16}$ Evidence of morphological and functional sporting differences between the lead and trail legs have been reported in fencers. Morphologically, greater cross-sectional area of the thigh of the fencer's lead leg has been reported. ${ }^{17,18}$ Electromyographic studies have demonstrated that the trail hip, knee and ankle extensor muscles are concentrically predominant during the propulsive phase of lunging movements, whereas the lead leg extensors are eccentrically predominant during the landing phase of lunging movements. ${ }^{19} \mathrm{Ny}-$ strom and colleagues reported greater isometric and isokinetic strength for the fencer's lead leg; ${ }^{17}$ however, more recent evidence using a larger sample size of national-level fencers did not identify a significant difference between the lead and trail legs for isokinetic knee extension strength. ${ }^{20}$

Evaluations of dynamic balance performance in fencers also appear to be divided regarding the presence of asymmetries between the lead and trail legs. Consistent with the approach used for athletes in other sports, recent studies evaluating dynamic balance in fencers have used the SEBT. ${ }^{14,15}$ The SEBT requires the athlete to perform a series of single leg squats while reaching in 8 different directions (anterior, anterolateral, lateral, posterolateral, posterior, posteromedial, medial, anteromedial) with their contralateral lower extremity. ${ }^{2}$ The reach distance of the contralateral lower extremity is used as a measure of dynamic balance proficiency. Guan and colleagues noted greater reaching distance in the posterolateral direction for the lead-leg of child fencers. ${ }^{14}$ Conversely, baseline measures of dynamic balance in club-level fencers between the ages of 14 and 35 did not reveal any differences between the lead and trail legs. ${ }^{15}$ To date there has not been an evaluation of dynamic balance performance in a group of elite fencers.

The primary objective of this investigation was to evaluate whether asymmetry of dynamic balance is present in uninjured national-level fencers. Given the inherent sporting asymmetry of fencing, it was hypothesized that elite fencers would demonstrate superior dynamic balance on the lead-leg of their fencing stance. A second a posteriori objective was to compare dynamic balance proficiency in elite fencers to that of other high-level athletes found in other studies. This may have implications for fencing-specific considerations when designing balance training programs or adapting those that were previously developed for athletes of other sports. For example, the proprioceptive training program implemented by de Vasconcelos and colleagues was adapted from a program originally developed for athletes having sustained ankle sprains. ${ }^{21}$ 


\section{MATERIALS AND METHODS}

\section{PARTICIPANTS}

A convenience sample of healthy adults aged 18-32 years was recruited for this study from a pool of elite fencing athletes in Canada. To be included in this study, participants were Canadian fencers, regardless of sex or weapon (sabre, foil, épée), who were competing and had been nationally ranked within the top-16 at any time between the 2016-2019 competition seasons. These inclusion criteria were set to justify an appropriate level of prior training and proficiency. All participants were at least 18 years of age on the date of data collection. Participants were excluded if they were experiencing, or had a history of, musculoskeletal injury to their lower extremities, or pelvis during the three months prior to data collection. This study was approved by the Canadian Memorial Chiropractic College's Research Ethics Board (REB \#1810B04). All participants provided written informed consent.

\section{EXPERIMENTAL PROTOCOL}

Once consent was received, participants' demographic and injury history were collected to confirm eligibility. Demographics included sex, height, mass and competitive fencing stance to identify the lead and trail legs.

Athletic attire (shorts and t-shirt) was worn by participants during the data collection session. First, the participant was asked to lie supine on a treatment table. The length of each lower limb was measured as the distance from the anterior superior iliac spine to the medial malleolus of the ankle using a tape measure. ${ }^{22}$

Participants then observed a video that provided a set of standardized instructions and demonstration for how to perform the YBT. ${ }^{23}$ The YBT is based on a subset of reach directions (anterior, posterolateral and posteromedial) that are part of the SEBT and has excellent intra-rater reliability $\left(\mathrm{ICC}_{3,1}=0.85-0.89\right) .{ }^{3}$ A YBT trial was defined as a single exertion in one of the three directions. Similarly, a YBT repetition was defined as a complete set of measurements in all three directions for a given lower extremity. All YBT trials were performed with the participant standing unshod on a commercially available apparatus (Y-Balance Test Kit). A research assistant used markings on the YBT apparatus to determine the reach distance to the nearest half-centimeter upon completion of each trial, and manually recorded each measurement on a data collection sheet. The same research assistant was used for all data collections.

First, participants completed six repetitions of the YBT on both their lead and trail legs as practice to mitigate a potential learning effect. ${ }^{24}$ After completing the six practice repetitions per leg (12 total repetitions), participants completed a total of three experimental repetitions of the YBT standing on both their lead and trail legs. Thus, participants completed a total of nine repetitions of the YBT on each of their lead and trail legs for a total 54 trials ([6 practice +3 experimental] * 2 legs * 3 directions). The order of the starting stance leg (lead or trail) was randomly determined for each participant. Each repetition of the YBT was performed in the order of anterior, posteromedial and posterolateral. Participants were given a minimum of 15 -seconds rest be- tween exertions with additional rest time provided upon request. To further mitigate against fatigue, execution of the YBT was performed on alternating legs after each repetition.

\section{DATA HANDLING AND REDUCTION}

Written measurements of reach distance from each YBT trial for each participant were transcribed into an electronic spreadsheet. This was completed using a double data entry method. Transcription was independently completed by a research assistant and an investigator who then met to resolve discrepant entries by reviewing the data collection sheet and coming to a consensus.

The average reach distance for the anterior, posteromedial and posterolateral trials of each YBT repetition were used to calculate a composite score. Thus, each repetition of the YBT was represented by four measurements. An average across the three experimental YBT repetitions for each of the four measurements was determined for the lead-leg of each participant. The same was done for the trail-leg. This resulted in a single value for each of the four reach distances (anterior, posteromedial, posterolateral and composite) for the lead and trail legs. Finally, participant leg lengths were used to normalize the reach distances. ${ }^{7}$ Normalized reach distances were expressed as a percentage for subsequent statistical analysis.

\section{STATISTICAL ANALYSIS}

All statistical analyses were performed using Excel (Microsoft, Redmond, WA, USA). Sample means, standard deviations, and 95\% confidence interval limits for the anterior, posteromedial, posterolateral and composite measures were derived as descriptive statistics. To address the primary objective, differences in reach distances along each direction and the composite measure between the lead and trail legs were statistically evaluated using one-tailed paired t-tests. An alpha level of 0.05 was used for all statistical analyses. Male and female YBT data from Division I collegiate athletes competing in different sports (basketball, golf, hockey, soccer, volleyball, football and wrestling) were used to construct $95 \%$ confidence interval limits for a qualitative comparison against dynamic balance proficiency of the fencers. ${ }^{25}$

\section{RESULTS}

Data were collected from 14 fencers. Demographics are reported in Table 1.

There were no statistically significant differences in normalized reach distances between the dominant and nondominant lower extremities (Table $2, p \geqslant 0.65$ ).

The confidence interval for the normalized reach distances in the anterior direction for the fencers at least partially overlapped the confidence intervals for collegiate athletes competing in different sports (Figure 1A). The lower limit of the confidence interval for the normalized reach distance in the posterolateral direction for the fencers was greater than the upper limits of the confidence intervals for collegiate athletes competing in different sports (Figure 
Table 1. Demographics of sample population. Values for age, height and mass are the group means with standard deviations reported in parentheses.

\begin{tabular}{|l|l|}
\hline Participants & $\mathrm{n}=14$ (8 female) \\
\hline Age (years) & $25.8(4.4)$ \\
\hline Height $(\mathrm{m})$ & $1.75(0.08)$ \\
\hline Mass $(\mathrm{kg})$ & $70.5(11.2)$ \\
\hline Lead Leg & 13 Right; 1 Left \\
\hline Lead Leg Length $(\mathrm{m})$ & $0.93(0.06)$ \\
\hline Trail Leg Length $(\mathrm{m})$ & $0.93(0.06)$ \\
\hline Weapon & Foil:6; Epee:8 \\
\hline
\end{tabular}

Table 2. Normalized YBT reach distances for the lead and trail legs in each direction and the composite score. All values are reported as a percentage of leg length.

\begin{tabular}{|c|c|c|c|}
\hline & & Mean (SD) (\%) & 95\% Cl (\%) \\
\hline \multirow{3}{*}{ Anterior } & Lead & $67(7)$ & $(63,70)$ \\
\cline { 2 - 4 } & Trail & $66(8)$ & $(62,70)$ \\
\hline \multirow{2}{*}{ Posterolateral } & Lead & $108(5)$ & $(105,111)$ \\
\cline { 2 - 4 } & Trail & $108(7)$ & $(109,111)$ \\
\hline \multirow{2}{*}{ Posteromedial } & Lead & $112(4)$ & $(109,114)$ \\
\cline { 2 - 4 } & Trail & $111(5)$ & $(92,98)$ \\
\hline \multirow{2}{*}{ Composite } & Lead & $95(6)$ & $(92,98)$ \\
\cline { 2 - 4 } & Trail & $95(5)$ & \\
\hline
\end{tabular}

$\mathrm{SD}=$ standard deviation $; \mathrm{CI}=$ confidence interval.

1B). Fencers also appeared to demonstrate enhanced dynamic balance in the posteromedial direction compared to collegiate athletes competing in basketball, golf, soccer, volleyball and football (Figure 1C). The greater normalized reach distances in the posteromedial and posterolateral directions contributed to better composite scores in fencers (Figure 1D).

\section{DISCUSSION}

The present study used the YBT to compare dynamic balance performance between the lead and trail legs of healthy elite fencers. A secondary objective sought to compare YBT performance between elite fencers and Division I collegiate athletes competing in different sports. Given the asymmetrical nature of fencing, it was hypothesized that fencers would demonstrate differences in dynamic balance between limbs. However, the results of the present study showed no statistically significant differences in YBT performance between the lead and trail legs. This indicates that dynamic balance between the two limbs in a group of healthy elite fencers are not significantly different, despite the inherent asymmetry of the sport. These data also showed that fencers may display greater reach distances in the posterolateral and posteromedial directions than other athletes.

Historically, research evaluating balance in fencers has focused on their control of upright standing posture. ${ }^{12,13}$ This largely ignores the ability of a fencer to control their balance while performing volitional movements. Two recent studies have used the SEBT, from which the YBT is derived, to evaluate dynamic balance performance in child and club-level fencers. ${ }^{14,15}$ Their authors reported conflicting findings regarding the presence of dynamic balance asymmetry in their participants. The current results were similar to those of de Vasconcelos and colleagues in that there was no observed difference in reach distance between the dominant and non-dominant lower extremities. ${ }^{15}$ Conversely, Guan and colleagues identified a side-to-side difference in the posterolateral direction, but their statistically significant findings were derived from combined data from fencers and Tae Kwon Do athletes. ${ }^{14}$ One possible explanation for the discrepant findings could be due to the chosen study population. Guan and colleagues studied child fencers with limited experience as their study population, whereas the current study and that of de Vasconcelos and colleagues focused on more experienced and likely more proficient fencers. ${ }^{14,15}$ Anecdotally, more experienced, and more proficient fencers will supplement their fencing training with general strength and conditioning training that may counteract their inherent potential sporting asymmetry. The results of the current study suggest that assessing dynamic balance performance in uninjured elite fencers may contribute to developing training regimens or monitoring 


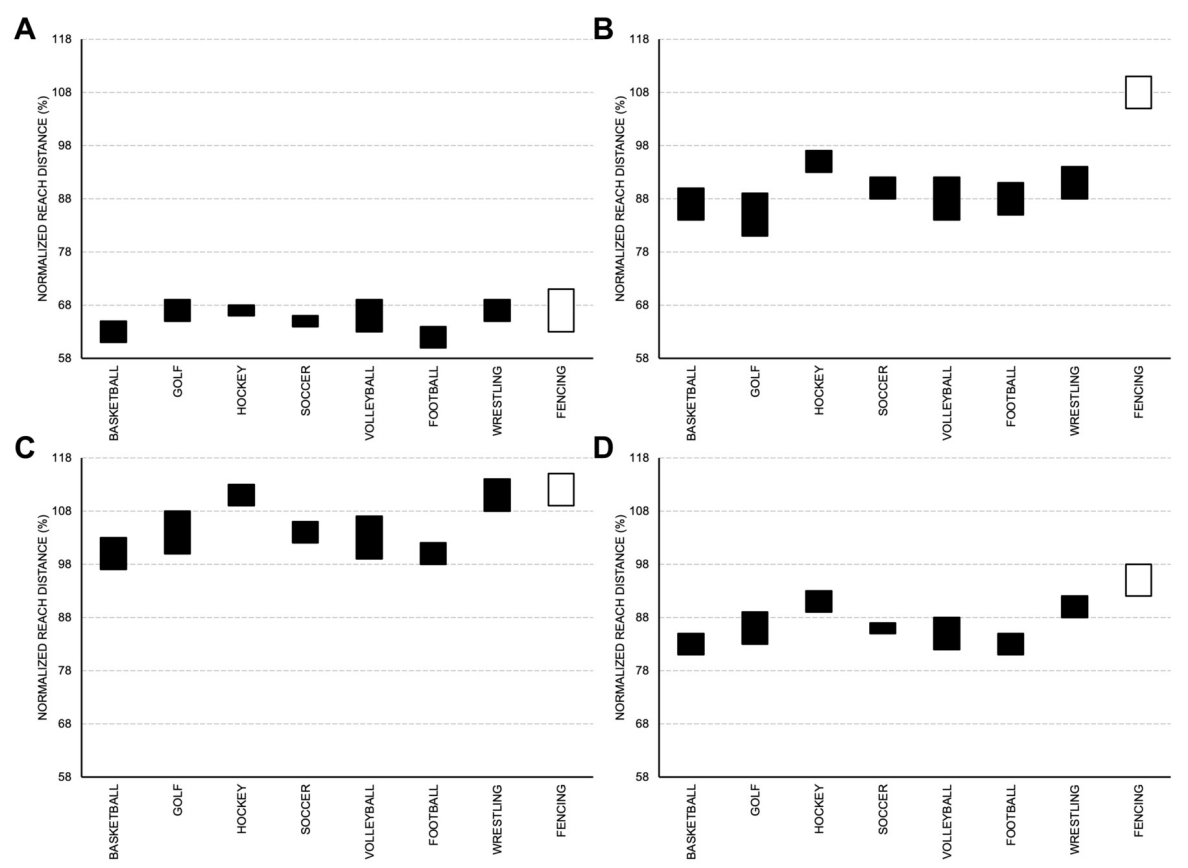

Figure 1. 95\% confidence intervals for normalized YBT reach distances of fencers (white bars) and Division I athletes (black bars; Stiffler et al., 2015) in the anterior (A), posterolateral (B), posteromedial (C) directions along with the composite score (D).

progress during rehabilitation when asymmetries are present. It also indicates that the morphological and functional asymmetries that have been previously documented in the lower extremities of fencers may not manifest as asymmetries in dynamic balance control; ${ }^{17,18}$ however, this statement must be tempered by the fact that the morphology and lower extremity strength of the fencers in this study was not measured.

Balance training has previously been advocated to aid fencers' performance. ${ }^{10,11}$ Data presented by de Vasconcelos and colleagues demonstrated that overall dynamic balance improved in fencers after a 12 -week proprioceptive training program that was adapted from one developed for athletes with ankle sprains. ${ }^{15}$ To the authors' knowledge, this has been the only study to date that has implemented a balance training program for fencers. Thus, an approach to developing sport-specific balance training programs might be to compare dynamic balance performance between fencers and athletes competing in other sports. For example, the elite fencers in the current study generally demonstrated greater normalized reach distances in the posterolateral and posteromedial directions than American collegiate athletes who competed in a variety of sports. ${ }^{25}$ This may be related to the similarity of movement patterns between the posterolateral and posteromedial directions in the YBT and the lunge that is commonly used in fencing. The extent to which lunge-specific training affects YBT reach distances in the posterolateral and posteromedial directions is currently unknown. Furthermore, the relationship between dynamic balance performance and fencing proficiency is also unknown, but greater lunge distance has been observed in elite fencers when compared to their novice counterparts. ${ }^{26}$

There were several limitations of the current study. Although data were obtained from male and female fencers, a sex-specific hypothesis was not formulated and subsequently was not addressed in statistical analyses. This was mainly due to constraints imposed on the sample size by inclusion/exclusion criteria and recruitment efforts. Previous work has demonstrated differences in kinematic strategies between males and females that resulted in greater normalized reach distances in females. ${ }^{27}$ No information was obtained regarding the current national ranking of the fencers who participated. Instead, the authors confirmed that the participant had achieved a top-16 national ranking in a three-year period prior to data collection. This decision was made to increase the size of the study population in consideration of the prevalence of lower extremity injuries in fencers and our exclusion criteria of an injury to either lower extremity or the pelvis in the three months prior to data collection. Finally, kinematic and kinetic data were not obtained in this study. These data could have provided information regarding local differences (e.g. joint angle) in movement strategy that may have left the global outcome (i.e. reach distance) unchanged.

\section{CONCLUSION}

Despite the asymmetrical nature of fencing, the results of the current study indicate that the dynamic balance performance of these elite healthy fencers was not statistically significantly different between their lead and trail legs. An $a$ posteriori analysis showed that normalized reach distances of this population were likely to have been greater than 
other elite athletes in the posterolateral and posteromedial directions which may be attributed to their training, though further studies are required to confirm these observations. The apparent symmetry of dynamic balance control in noninjured fencers indicates that the YBT may be used in this population during training, rehabilitation, and assessment.

\section{CONFLICT OF INTEREST $\{\#$ COI $\}$}

None to declare.

Submitted: March 29, 2021 CST, Accepted: October 19, 2021 CST 


\section{REFERENCES}

1. Hrysomallis C. Balance ability and athletic performance. Sport Med. 2011;41(3):221-232. doi:10.2 $\underline{165 / 11538560-000000000-00000}$

2. Gribble PA, Hertel J, Plisky P. Using the star excursion balance test to assess dynamic posturalcontrol deficits and outcomes in lower extremity injury: A literature and systematic review. J Athl Train. 2012;47(3):339-357. doi:10.4085/1062-6050-4 $\underline{7.3 .08}$

3. Plisky PJ, Gorman PP, Butler RJ, Kiesel KB, Underwood FB, Elkins B. The reliability of an instrumented device for measuring components of the star excursion balance test. N Am J Sports Phys Ther. 2009;4(2):92-99.

4. Grassi A, Alexiou K, Amendola A, et al. Postural stability deficit could predict ankle sprains: a systematic review. Knee Surgery, Sport Traumatol Arthrosc. 2018;26(10):3140-3155. doi:10.1007/s0016 $\underline{7-017-4818-\mathrm{X}}$

5. De Noronha M, França LC, Haupenthal A, Nunes GS. Intrinsic predictive factors for ankle sprain in active university students: A prospective study. Scand J Med Sci Sport. 2013;23(5):541-547. doi:10.1111/j.160 0-0838.2011.01434.X

6. Hertel J, Braham RA, Hale SA, Olmsted-Kramer LC. Simplifying the star excursion balance test: Analyses of subjects with and without chronic ankle instability. J Orthop Sports Phys Ther. 2006;36(3):131-137. doi:1 0.2519/jospt.2006.36.3.131

7. Plisky PJ, Rauh MJ, Kaminski TW, Underwood FB. Star excursion balance test as a predictor of lower extremity injury in high school basketball players. $J$ Orthop Sports Phys Ther. 2006;36(12):911-919. doi:1 0.2519/jospt.2006.2244

8. Butler RJ, Lehr ME, Fink ML, Kiesel KB, Plisky PJ. Dynamic Balance Performance and Noncontact Lower Extremity Injury in College Football Players: An Initial Study. Sports Health. 2013;5(5):417-422. doi:1 $\underline{0.1177 / 1941738113498703}$

9. Gonell AC, Romero JAP, Soler LM. Relationship between the Y-balance test scores and soft tissue injury incidence in a soccer team. Int J Sports Phys Ther. 2015;10(7):955-966.

10. Chen TLW, Wong DWC, Wang Y, Ren S, Yan F, Zhang M. Biomechanics of fencing sport: A scoping review. PLOS ONE. 2017;12(2):1-22. doi:10.1371/journ al.pone. 0171578
11. Harmer PA. Epidemiology of time-loss injuries in international fencing: A prospective, 5-year analysis of Fédération Internationale d'Escrime competitions. Br J Sports Med. 2019;53(7):442-448. doi:10.1136/bjsp orts-2018-100002

12. Herpin G, Gauchard GC, Lion A, Collet P, Keller D, Perrin PP. Sensorimotor specificities in balance control of expert fencers and pistol shooters. $J$ Electromyogr Kinesiol. 2010;20(1):162-169. doi:10.101 6/j.jelekin.2009.01.003

13. Kim T, Kil S, Chung J, Moon J, Oh E. Effects of specific muscle imbalance improvement training on the balance ability in elite fencers. J Phys Ther Sci. 2015;27(5):1589-1592. doi:10.1589/jpts.27.1589

14. Guan Y, Bredin S, Taunton J, et al. Bilateral difference between lower limbs in children practicing laterally dominant vs. non-laterally dominant sports. Eur J Sport Sci. 2020:1-23. doi:10.1080/17461391.202 0.1814425

15. de Vasconcelos GS, Cini A, Lima CS. Proprioceptive training on dynamic neuromuscular control in fencers: A clinical trial. J Sport Rehabil. 2020:1-6. doi:10.1123/jsr.2019-0469

16. Maloney SJ. The relationship between asymmetry and athletic performance: A critical review. J Strength Cond Res. 2019;33(9):2579-2593. doi:10.1519/ISC.000 $\underline{0000000002608}$

17. Nystrom J, Lindwall O, Ceci R, Harmenberg J, Swedenhag J, Ekblom B. Physiological and morphological characteristics of world class fencers. Int J Sports Med. 1990;11(2):136-139. doi:10.1055/s-2 007-1024778

18. Tsolakis BV. Anthropometric profile and limb asymmetries in young male and female fencers. J Hum Mov Stud. 2006;50:201-216.

19. Guilhem G, Giroux C, Couturier A, Chollet D, Rabita G. Mechanical and muscular coordination patterns during a high-level fencing assault. Med Sci Sports Exerc. 2014;46(2):341-350. doi:10.1249/MSS.0b $\underline{013 \mathrm{e} 3182 \mathrm{a} 6401 \mathrm{~b}}$

20. Poulis I, Chatzis S, Christopoulou K, Tsolakis Ch. Isokinetic strength during knee flexion and extension in elite fencers. Percept Mot Skills. 2009;108(3):949-961. doi:10.2466/PMS.108.3.949-961 
21. Hupperets MDW, Verhagen EALM, Van Mechelen W. Effect of unsupervised home based proprioceptive training on recurrences of ankle sprain: Randomised controlled trial. BMJ . 2009;339:b2684. doi:10.1136/b mj.b2684

22. Brady RJ, Dean JB, Skinner TM, Gross MT. Limb length inequality: Clinical implications for assessment and intervention. J Orthop Sports Phys Ther. 2003;33(5):221-234. doi:10.2519/jospt.2003.3 $\underline{3.5 .221}$

23. Scottish Rite for Children. (40) Y-Balance Testing - Scottish Rite for Children - YouTube. https://www.y outube.com/watch? $\mathrm{v}=0$ S65kbx2na0\&t=123s.

Published December 15, 2016. Accessed July 9, 2020.

24. Hertel J, Miller SJ, Denegar CR. Intratester and intertester reliability during the star excursion balance tests. J Sport Rehabil. 2000;9(2):104-116. do $\mathrm{i}: 10.1123$ /jsr.9.2.104
25. Stiffler MR, Sanfilippo JL, Brooks MA, Heiderscheit BC. Star excursion balance test performance varies by sport in healthy division $\mathrm{i}$ collegiate athletes. J Orthop Sports Phys Ther. 2015;45(10):772-780. doi:10.2519/jospt.2015.5777

26. Mulloy F, Mullineaux DR, Irwin G. Use of the kinematic chain in the fencing attacking lunge. In: Proceedings of the 33rd International Conference on Biomechanics in Sports. Poitiers, France; 2015.

27. Gribble PA, Robinson RH, Hertel J, Denegar CR. The effects of gender and fatigue on dynamic postural control. J Sport Rehabil. 2009;18(2):240-257. doi:10.11 23/jsr.18.2.240 